\title{
Optimal Cost Control for Variable Sampling Period Network Control System with Actuator Failure
}

\author{
Ling Wang, Nan Xie* \\ College of Computer Science and Technology, Shandong University of Technology, Zibo, China
}

Email address:

wangling1991@outlook.com (Ling Wang), xienan@sdut.edu.cn (Nan Xie)

${ }^{*}$ Corresponding author

\section{To cite this article:}

Ling Wang, Nan Xie. Optimal Cost Control for Variable Sampling Period Network Control System with Actuator Failure. Automation, Control and Intelligent Systems. Vol. 4, No. 4, 2016, pp. 66-72. doi: 10.11648/j.acis.20160404.11

Received: July 18, 2016; Accepted: July 28, 2016; Published: August 17, 2016

\begin{abstract}
This paper considers the networked control systems (NCSs) with the varying sampling period and actuator failure. When the NCSs are modeled, the varying sampling period was described by a constant sampling period and a network delay. Base on this and the actuator failed, using the Lyapunov stability theory and linear matrix inequalities to prove the existence of the cost guaranteed performance, and obtain the optimal cost guaranteed performance controller.
\end{abstract}

Keywords: Network Control System, Varying Sampling Period, Delay, Actuator Failure, Optimal Cost Guaranteed Performance

\section{Introduction}

Network control system (NCS) is a distributed closed-loop feedback control system which is composed of sensors, controllers and actuators [1]. The controller exchanges the information with sensor and actuator according to the internet [2]. Compare to the traditional control system, the NCS not only can share the information but also can use the remote operate and control. So the NCS become more and more popular. But also because of the use of the internet in the NCS, the NCS has some problems, such as delay, data packet dropout.

In the NCS commonly use continuous controlled objects and discrete controllers. So we can regard it as a sampling system to study [3]. The sensor reads information for a period of time. The time interval is called the sampling period. The selection of the sampling period can impact system performance [4]. So the study of the variable sampling network control system has become meaningful. And if the actor broke down the NCS will can't work properly. And it will lead to a huge lose [5]. So we can see the importance of the fault-tolerant of NCS [6-8].

In recent years, in order to deal with the sampling period problem most study assuming that the sampling period is a constant [9]. Actually the sampling period is unstable. Yi
Jianqiang used the delay to represent the sampling period [10]. Xie Guangming translated the variable sampling period into the uncertainty of system parameters [11]. Yu-Long Wang assumed that the sampling period can chose a random value in a finite set [12]. For the fault tolerant problem, Li Wei used the switch matrix presents the actuator's state [13]. But it just can present the actuator in the normal working state or the actuator complete failure. It can't describe the actuator partial failure. Some people also discard the wrong data and still use the last period's data. But it can't effectively improve the performance of the system.

Fan Jinrong provided a function to deal with the sampling period. Sampling period described by the delay and a number what is continuous changed in a limited range [9]. And this paper will base on that to do the further study. And in order to deal with the actuator failure problem we refer to Li Yu's method. In that function not only can describe the normal case and outage case but also can describe the actuator partial degradation [14]. First we will introduce Fan Jinrong's function to deal with the variable sampling period. It is described as follows

Symbol description: The symbol * indicates the block matrix in a symmetric matrix, $A^{\mathrm{T}}$ is the transpose matrix of $A$. 


\section{Problem Description and Preparation}

Consider the network control system describes by the Figure 1, controlled object is a linear time invariant system, and it is described by the following state equation:

$$
P:\left\{\begin{array}{l}
\dot{x}(t)=A_{c} x(t)+B_{c} u(t) \\
y(t)=C_{c} x(t)
\end{array}\right.
$$

where $x(t), \mathrm{u}(t)$ and $y(t)$ represent the input state, control input and output state respectively.

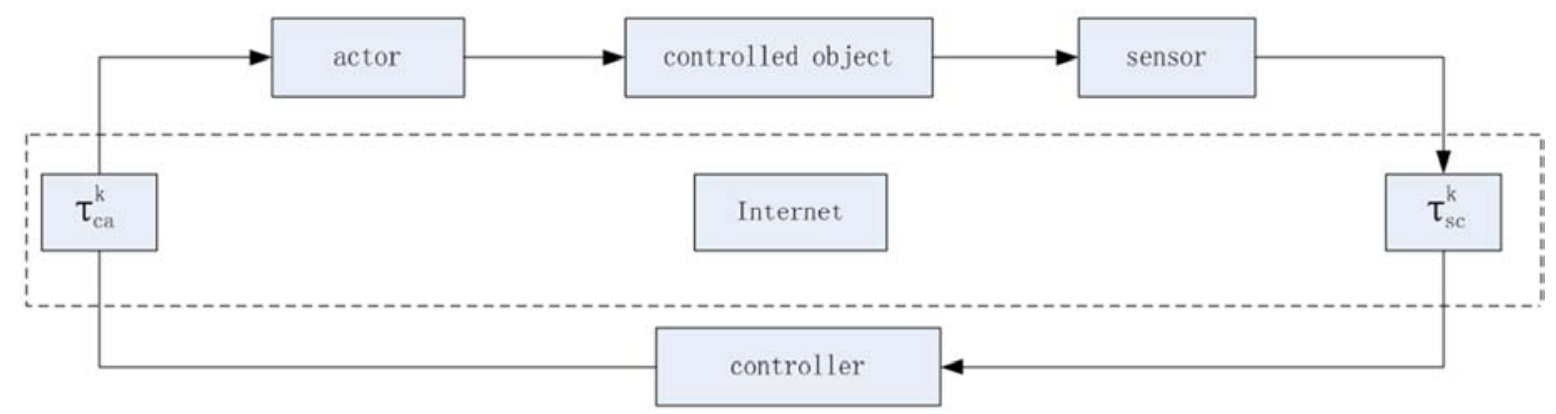

Figure 1. A simplified network control system.

This article uses the static memory-less state feedback controller, so controller can be moved to the actuator side without affecting the performance of the system. Therefore the time delay from the sensor to the controller $\tau_{s c}^{k}$, the time delay from controller to the actuator $\tau_{s c}^{k}$ and the time delay for the controller to the calculate $\tau_{c}^{k}$ can be combined together, we can regard $\tau_{k}=\tau_{s c}^{k}+\tau_{c a}^{k}+\tau_{c}^{k}=\tau$ as a constant. In order to facilitate analysis, highlight the characteristics of variable sampling period network control system, the system to do the following assumptions:

(1) Just think about short time delay, where $\tau<h_{\min }$.

(2) Time-varying sampling period $T_{k}=h_{k}+\tau, h_{k}$ is time-varying and bounded, so the sampling period is time-varying and bounded $T_{k} \in\left[T_{\min }, T_{\max }\right]$.

(3) The nominal value of $h_{k}$ is $h_{0}$, so $h_{k}=h_{0}+\Delta k$, where $\Delta k$ represents the uncertain part of variable sampling period.

(4) The sensor in this system is time-driven, sampling instant is $t_{k}$ and sampling period is $T_{k}=t_{k+1}-t_{k}$. The controller and the actuator are event-driven.

(5) The input state $u\left(t-\tau_{k}\right)$ remains unchanged during the time $t \in\left[t_{k}, t_{k}+\tau\right]$ and $t \in\left[t_{k}+\tau, t_{k+1}\right]$, and does not synchronously change with sensors in the sampling time $t_{k}$ owning to the actuator with zero-order holder. So

$$
u(t)=\left\{\begin{array}{l}
u\left(t_{k-1}\right), t_{k} \leq t<t_{k}+\tau \\
u\left(t_{k}\right), t_{k}+\tau \leq t<t_{k+1}
\end{array}\right.
$$

According to the sampling period $h_{k}$ to discretize the controlled object, we can get the discrete state equation of controlled object:

$$
\begin{aligned}
x(k+1) & =e^{A\left(h_{k}+\tau\right)} x(k)+\int_{k T_{k}}^{k T_{k}+\tau} e^{A_{c}\left(k T_{k}+T-s\right)} d s B_{c} u(k-1) \\
& +\int_{k T_{k}+\tau}^{k T_{k}+T_{k}} e^{A_{c}\left(k T_{k}+T-s\right)} d s B_{c} u(k)=e^{A\left(h_{k}+\tau\right)} x(k) \\
& +e^{A_{c} h_{k}} \int_{0}^{\tau} e^{A_{c} s} d s B_{c} u(k-1)+\int_{0}^{h_{k}} e^{A_{c} s} d s B_{c} u(k)
\end{aligned}
$$

According to $H=\left[\begin{array}{cc}A_{c} & B_{c} \\ 0 & 0\end{array}\right]$ where $A_{c}$ and $B_{c}$ are constant matrices of appropriate dimensions, there is

$$
F(T) \triangleq \exp (H T)=\left[\begin{array}{cc}
\exp \left(A_{c} T\right) & \int_{0}^{T} \exp \left(A_{c} s\right) B_{c} d s \\
0 & I
\end{array}\right]
$$

Introducing augmented variables $z(k)=\left[x^{\mathrm{T}}(k) \mathrm{u}^{\mathrm{T}}(\mathrm{k}-1)\right]^{\mathrm{T}}$, (3) can be written as

$$
z(k+1)=\Phi\left(h_{k}\right) z(k)+\Gamma\left(h_{k}\right) u(k)
$$

where

$$
\begin{aligned}
& \Phi\left(h_{k}\right)=\left[\begin{array}{cc}
e^{A_{c}\left(h_{k}+\tau\right)} & e^{A_{c} h_{k}} \int_{0}^{\tau} e^{A_{c} s} d s B_{c} \\
0 & 0
\end{array}\right] \\
& =A_{0}+\left[\begin{array}{l}
I \\
0
\end{array}\right]\left[\begin{array}{ll}
e^{A_{c} \Delta k}-I & \int_{0}^{\Delta k} e^{A_{c} s} B_{c} d s
\end{array}\right] A_{0} \\
& =A_{0}+\beta_{T}^{-1}\left[\begin{array}{l}
I \\
0
\end{array}\right] \beta_{T} \int_{0}^{\Delta k} e^{A_{c} s} d s\left[\begin{array}{ll}
A_{c} & B_{c}
\end{array}\right] A_{0} \\
& =A_{0}+D F_{K} E_{1} \\
& \Gamma\left(h_{k}\right)=\int_{0}^{h_{k}} e^{A_{c} s} d s B_{c} u(k)
\end{aligned}
$$

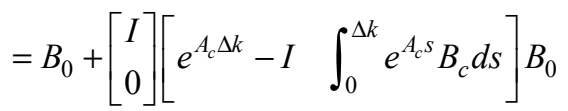

$$
\begin{aligned}
& =B_{0}+D F_{k} E_{2}
\end{aligned}
$$




$$
A_{0}=\left[\begin{array}{cc}
e^{A_{c}\left(h_{0}+\tau\right)} & e^{A_{c} h_{0}} \int_{0}^{\tau} e^{A_{c} s} d s B_{c} \\
0 & 0
\end{array}\right]=F\left(h_{0}\right)\left[\begin{array}{ll}
I & 0 \\
0 & 0
\end{array}\right] F(\tau),
$$$$
B_{0}=\left[\begin{array}{c}
\int_{0}^{h_{0}} e^{A_{c} s} d s B_{c} \\
I
\end{array}\right]=F\left(h_{0}\right)\left[\begin{array}{l}
I \\
0
\end{array}\right], D=\beta_{T}^{-1}\left[\begin{array}{l}
I \\
0
\end{array}\right] \text {, }
$$

$F_{k}=\beta_{T} \int_{0}^{\Delta k} e^{A_{c} s} d s, E_{1}=\left[\begin{array}{ll}A_{c} & B_{c}\end{array}\right] A_{0}, E_{2}=\left[\begin{array}{ll}A_{c} & B_{c}\end{array}\right] B_{0}$,

$\beta_{T}$ is a required value.

The system (1) can be described by the following state equation:

$$
\left\{\begin{array}{l}
z(k+1)=\left(A_{0}+D F_{k} E_{1}\right) z(k)+\left(B_{0}+D F_{k} E_{2}\right) u(k) \\
y(k)=C z(k)
\end{array}\right.
$$

where

$$
C=\left[C_{c}, 0\right], F_{k}^{\mathrm{T}} F_{K} \leq I
$$

In order to deal the actuator failure, we refer the function that is producted by $\mathrm{Li} \mathrm{Yu}$ [14]. And it is described as the follows.

For control input $u_{i}, i=1,2 \cdots m$, let $u_{i}^{\mathrm{F}}$ denotes the signal from the actuator that has failed. The following failure model is adopted in this paper

$$
u_{i}^{\mathrm{F}}=\alpha_{i} u_{i}, i=1,2 \cdots m
$$

where $0 \leq \widehat{\alpha}_{i} \leq \alpha_{i} \leq \breve{\alpha}_{i}, i=1,2 \cdots m$ with $\hat{\alpha}_{i} \leq 1, \breve{\alpha}_{i} \geq 1$

Define the $\alpha$, that is described as follows:

In the above model of actuator failure, if $\breve{\alpha}_{i}=\widehat{\alpha}_{i}$, then it corresponds to the normal case $u_{i}^{\mathrm{F}}=u_{i}$; When $\breve{\alpha}_{i}=0$, it covers the outage case. If $\hat{\alpha}_{i}>0$, it corresponds to the partial failure case, i.e., partial degradation of the actuator.

Denote:

$$
\begin{aligned}
& u^{\mathrm{F}}=\left[u_{1}^{\mathrm{F}}, u_{2}^{\mathrm{F}}, \cdots u_{m}^{\mathrm{F}}\right]^{\mathrm{T}} \\
& \breve{\alpha}=\operatorname{diag}\left\{\breve{\alpha}_{1}, \breve{\alpha}_{2}, \cdots \breve{\alpha}_{m}\right\} \\
& \widehat{\alpha}=\operatorname{diag}\left\{\widehat{\alpha}_{1}, \widehat{\alpha}_{2}, \cdots \widehat{\alpha}_{m}\right\} \\
& \alpha=\operatorname{diag}\left\{\alpha_{1}, \alpha_{2}, \cdots \alpha_{m}\right\}
\end{aligned}
$$

$\alpha$ is said to be admissible if $\alpha$ satisfies $\hat{\alpha} \leq \alpha \leq \breve{\alpha}$.

So (8) can be represented by

$$
\mathrm{z}(k+1)=A z(k)+B \alpha u(k)
$$

where

$$
A=A_{0}+D F_{k} E_{1}, B=B_{0}+D F_{k} E_{2}
$$

For system (12) denote a cost function

$$
J=\sum_{k=0}^{\infty}\left[z^{T}(k) Q z(k)+u^{T}(k) \alpha^{T} R \alpha u(k)\right]
$$

where $Q>0$ and $R>0$ are given weighting matrices.

Definition 1: For the uncertain system (12) and cost function (14), if there exist a matrix $K$ and a positive number $J^{*}$ such that the close-loop NCS is stable and cost function satisfies $J \leq J^{*}$, then $u(k)=K z(k)$ is said to be a guaranteed cost control law and $J^{*}$ is the upper bound of quadratic performance.

Lemma 1 [15]: Set $\phi$ is any square, if exist matrix $P=X^{-1}$ such that $\phi^{\mathrm{T}} P \phi-P+T<0$ if and only if there exist a matrix $X>0$ is satisfies $\left[\begin{array}{cc}-X & \phi X \\ * & -X+X T X\end{array}\right]<0$.

Lemma 2 [15]: If R, S and F are real matrices of appropriate dimensions, and $F_{k}^{\mathrm{T}} F_{K} \leq I$, then for any positive number $\varepsilon>0$, the following linear matrix inequality(LMI) satisfies $R F_{k} S+S^{\mathrm{T}} F_{k}^{\mathrm{T}} R^{\mathrm{T}} \leq \varepsilon R R^{\mathrm{T}}+\varepsilon^{-1} S^{\mathrm{T}} S$.

Lemma 3 [16]: For a constant matrix $A_{C}$, if $\mathrm{t} \geq 0$, so $\left\|\mathrm{e}^{A_{c} t}\right\| \leq e^{\eta t}$.

Lemma 4 [3]: The system contains an uncertainty sampling period $F_{\mathrm{k}}$. And it is norm-bounded, $|\Delta \mathrm{k}| \in\left[0, \Delta_{m a k}\right]$, if the real number $\beta \neq 0$ and satisfy the following condition: $|\beta| \leq\left\{\begin{array}{l}\eta /\left(e^{\eta \Delta_{\max }}-1\right), \eta \neq 0 \\ 1 / \Delta_{\max }, \quad \eta=0\end{array}\right.$, so $F_{\mathrm{k}}^{T} F_{k} \leq I$,

where $\eta=\frac{1}{2} \sigma_{\max }\left(A_{C}+A_{C}^{*}\right), \sigma(\bullet)$ represents the maximum singular value, and $A_{\mathrm{c}}^{*}$ is the conjugate transpose matrix of $A_{\text {c. }}$.

\section{Main Results}

Theorem 1: If any feasible $\alpha$ and symmetric positive definite matrix $P, Q$ satisfy the following LMI

$$
(A+B \alpha K)^{\mathrm{T}} P(A+B \alpha K)-P+Q+(\alpha K)^{\mathrm{T}} R \alpha K<0
$$

then $u(k)=K z(k)$ is the guaranteed cost control of system (12) and the upper bound of quadratic performance is

$$
J^{*}=z^{\mathrm{T}}(0) P z(0)
$$

Proof: Take $u(k)=K z(k)$ in the system (12) and the cost function (14). Suppose now there exist symmetric positive definite matrices $P, Q$ such that matrix inequality (15) holds for all admissible uncertainties, then the Lyapunov function candidate $V(z)=z^{\mathrm{T}} P z$ is positive definite. The corresponding Lyapunov difference along any trajectory of the close-loop system (12) is given by 


$$
\begin{aligned}
\Delta V(z) & =V(z(k+1))-V(z(k))=z^{\mathrm{T}}(k+1) P z(k+1)-z^{\mathrm{T}}(k) P z(k) \\
& =[A z(k)+B \alpha u(k)]^{\mathrm{T}} P[A z(k)+B \alpha u(k)]-z^{\mathrm{T}}(k) P z(k) \\
& =[\mathrm{Az}(\mathrm{k})+\mathrm{B} \alpha K z(k)]^{\mathrm{T}} \mathrm{P}[\mathrm{Az}(\mathrm{k})+\mathrm{B} \alpha \mathrm{Kz}(\mathrm{k})]-z^{\mathrm{T}}(k) P z(k) \\
& =z^{\mathrm{T}}(k)\left[(A+B \alpha K)^{\mathrm{T}} P(A+B \alpha K)-P\right] z(k)
\end{aligned}
$$

From condition (15), we have $\Delta V<-z^{\mathrm{T}}(k)\left[Q+(\alpha K)^{\mathrm{T}} R \alpha K\right] z(k)$. It follows from Lyapunov stability theory that the system (12) is asymptotically stable.

Summing both sides of the above inequality from 0 to $\infty$, we can get that

$$
V(z(\infty))-V(z(0))<-\sum_{k=0}^{\infty} z^{\mathrm{T}}(k)\left[Q+(\alpha K)^{\mathrm{T}} R \alpha K\right] z(k)
$$

Use the system asymptotically stability and $J=\sum_{k=0}^{\infty} z^{\mathrm{T}}(k)\left[Q+(\alpha K)^{\mathrm{T}} R \alpha K\right] z(k)$ yield $-z^{\mathrm{T}}(0) P z(0)<-J$. It's equal to

$$
J<z^{\mathrm{T}}(0) P z(0)
$$

The upper bound of the system performance index what conclude from theorem 1 depends on the initial state $z_{0}$, if $z_{0}$ is a zero-mean random variable and satisfies $E\left(z_{0} z_{0}^{\mathrm{T}}\right)=I$, then system performance index satisfies:

(I)

$$
\left[\begin{array}{c}
\gamma D D^{\mathrm{T}}-X+B_{0} R_{0} B_{0}^{\mathrm{T}} \\
* \\
* \\
* \\
* \\
*
\end{array}\right.
$$$$
\begin{gathered}
A_{0} X+B_{0} \beta Y \\
-R_{0} \beta_{0}^{-2} \\
* \\
* \\
* \\
*
\end{gathered}
$$

(II)

$$
\left[\begin{array}{ll}
X & I \\
I & S
\end{array}\right]<0
$$

has a solution $\left(\tilde{\gamma}, \tilde{S}, \tilde{X}, \tilde{Y}, \tilde{R}_{0}\right)$, then $u(k)=K z(k)=\tilde{Y} \tilde{X}^{-1} z(k)$ is the optimal quadratic guaranteed cost control law for system (12) and the corresponding upper bound of the system performance index is

$$
J^{*}=\operatorname{Trace}\left(\tilde{X}^{-1}\right)
$$

Proof: In light of theorem 1, the uncertain system (12) exists an optimal guaranteed cost control law if and only if there exist matrix $K$, symmetric matrix $P>0$ and any feasible $\alpha$ satisfy that

$$
E\{J\} \leq E\left\{z_{0} P z_{0}^{\mathrm{T}}\right\}=\operatorname{Trace}(P)
$$

Define

$$
\beta=\operatorname{diag}\left\{\beta_{1}, \beta_{2} \cdots \beta_{m}\right\} \quad \text { and } \beta_{0}=\operatorname{diag}\left\{\beta_{10}, \beta_{20} \cdots \beta_{m 0}\right\}
$$

where

$$
\begin{gathered}
\beta i=\frac{\widehat{\alpha} i+\breve{\alpha} \mathrm{i}}{2} \quad \beta_{i 0}=\frac{\breve{\alpha}_{i}-\widehat{\alpha}_{\mathrm{i}}}{\breve{\alpha}_{i}+\widehat{\alpha}_{\mathrm{i}}} \quad \mathrm{i}=1,2 \cdots \mathrm{m} \\
\alpha=\left(I+\alpha_{0}\right) \beta \quad \text { and } \quad\left|\alpha_{0}\right| \leq \beta_{0} \leq I
\end{gathered}
$$

where

$$
\alpha_{0}=\operatorname{diag}\left\{\alpha_{01}, \alpha_{02}, \cdots \alpha_{0 m}\right\},\left|\alpha_{0}\right|=\operatorname{diag}\left\{\left|\alpha_{01}\right|,\left|\alpha_{02}\right|, \cdots\left|\alpha_{0 m}\right|\right\}
$$

Theorem 2: Consider system (12) with cost function (14), if the following optimization problem

$$
\text { ST: } \min _{\gamma, X, S, Y, R_{0}} \operatorname{Trace}(S)
$$

$$
(A+B \alpha K)^{\mathrm{T}} P(A+B \alpha K)-P+Q+(\alpha K)^{\mathrm{T}} R \alpha K<0
$$

In light of Lemma 1, the inequality exists a matrix $X>0$ satisfies that

$$
\left[\begin{array}{cc}
-X & (A+B \alpha K) X \\
* & -X+X\left[Q+(\alpha K)^{\mathrm{T}} R \alpha K\right] X
\end{array}\right]<0
$$

where

$$
A=A_{0}+D F_{k} E_{1}, B=B_{0}+D F_{k} E_{2}
$$

Define a matrix

$$
M \stackrel{\Delta}{=}\left[\begin{array}{cc}
-X & \left(A_{0}+B_{0} \alpha K\right) X \\
* & -X+X\left[Q+(\alpha K)^{\mathrm{T}} R \alpha K\right] X
\end{array}\right]
$$


The inequality (29) can be written as

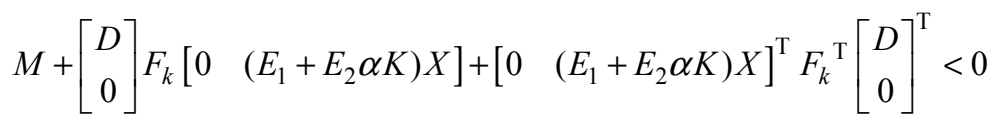

In light of Lemma 2: If the inequality (32) exists, if and only if there exists a constant $\gamma>0$, such that

$$
M+\gamma\left[\begin{array}{c}
D \\
0
\end{array}\right]\left[\begin{array}{ll}
D^{\mathrm{T}} & 0
\end{array}\right]+\gamma^{-1}\left[\begin{array}{c}
0 \\
X\left(E_{1}+E_{2} \alpha K\right)^{\mathrm{T}}
\end{array}\right]\left[\begin{array}{ll}
0 & \left.\left(E_{1}+E_{2} \alpha K\right) X\right]<0
\end{array}\right.
$$

take $M$ in the inequality, then

$$
\left[\begin{array}{cc}
\gamma D D^{\mathrm{T}}-X & \left(A_{0}+B_{0} \alpha K\right) X \\
* & -X+X\left[Q+(\alpha K)^{\mathrm{T}} R \alpha K\right] X+\gamma^{-1} X\left(E_{1}+E_{2} \alpha K\right)^{\mathrm{T}}\left(E_{1}+E_{2} \alpha K\right) X
\end{array}\right]<0
$$

It follows from the Schur complement that the above inequality is equivalent to

$$
\left[\begin{array}{ccc}
\gamma D D^{\mathrm{T}}-X & \left(A_{0}+B_{0} \alpha K\right) X & 0 \\
* & -X+X\left[Q+(\alpha K)^{\mathrm{T}} R \alpha K\right] X & X\left(E_{1}+E_{2} \alpha K\right)^{\mathrm{T}} \\
* & * & -\gamma I
\end{array}\right]<0
$$

It follows from the Schur complement that the above inequality is equivalent to

$$
\left[\begin{array}{ccccc}
\gamma D D^{\mathrm{T}}-X & \left(A_{0}+B_{0} \alpha K\right) X & 0 & 0 & 0 \\
* & -X & X\left(E_{1}+E_{2} K\right)^{\mathrm{T}} & X & (\alpha K X)^{\mathrm{T}} \\
* & * & -\gamma I & 0 & 0 \\
* & * & * & -Q^{-1} & 0 \\
* & * & * & * & -R^{-1}
\end{array}\right]<0
$$

Define $Y=K X$, we can get that

$$
\left[\begin{array}{ccccc}
\gamma D D^{\mathrm{T}}-X & A_{0} X+B_{0} \alpha Y & 0 & 0 & 0 \\
* & -X & \left(E_{1} X+E_{2} Y\right)^{\mathrm{T}} & X & (\alpha Y)^{\mathrm{T}} \\
* & * & -\gamma I & 0 & 0 \\
* & * & * & -Q^{-1} & 0 \\
* & * & * & * & -R^{-1}
\end{array}\right]<0
$$

Take (23) in the inequality

$$
\begin{aligned}
& {\left[\begin{array}{ccccc}
\gamma D D^{\mathrm{T}}-X & A_{0} X+B_{0}\left(I+\alpha_{0}\right) \beta Y & 0 & 0 & 0 \\
* & -X & \left(E_{1} X+E_{2} Y\right)^{\mathrm{T}} & X & {\left[\left(I+\alpha_{0}\right) \beta Y\right]^{\mathrm{T}}} \\
* & * & -\gamma I & 0 & 0 \\
* & * & * & -Q^{-1} & 0 \\
* & * & * & * & -R^{-1}
\end{array}\right]<0} \\
& =\left[\begin{array}{ccccc}
\gamma D D^{\mathrm{T}}-X & A_{0} X+B_{0} \beta Y & 0 & 0 & 0 \\
* & -X & \left(E_{1} X+E_{2} Y\right)^{\mathrm{T}} & X & (\beta Y)^{\mathrm{T}} \\
* & * & -\gamma I & 0 & 0 \\
* & * & * & -Q^{-1} & 0 \\
* & * & * & * & -R^{-1}
\end{array}\right]+\left[\begin{array}{ccccc}
0 & B_{0} \alpha_{0} \beta Y & 0 & 0 & 0 \\
* & 0 & 0 & 0 & \left(\alpha_{0} \beta Y\right)^{\mathrm{T}} \\
0 & 0 & 0 & 0 & 0 \\
0 & 0 & 0 & 0 & 0 \\
0 & * & 0 & 0 & 0
\end{array}\right]<0
\end{aligned}
$$




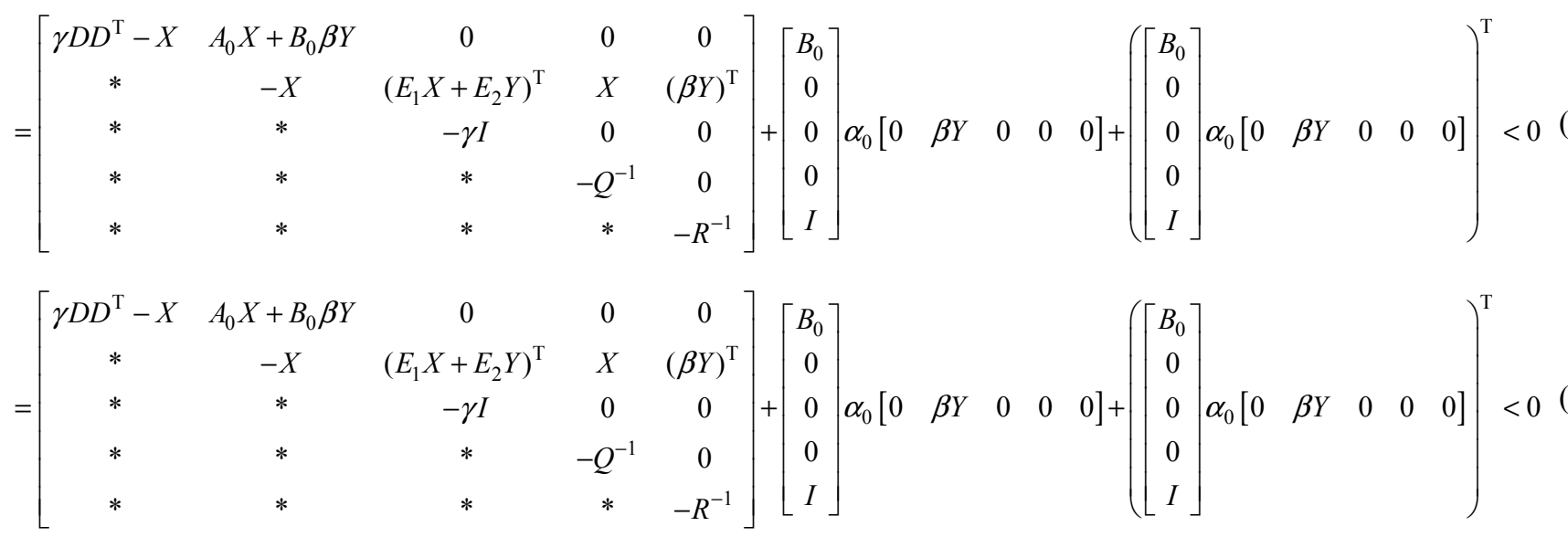

Using the inequality $2 a^{\mathrm{T}} b \leq a^{\mathrm{T}} a+b^{\mathrm{T}} b$ for any diagonal matrix $R_{0}>0$, it follows that

$$
\left[\begin{array}{ccccc}
\gamma D D^{\mathrm{T}}-X & A_{0} X+B_{0} \beta Y & 0 & 0 & 0 \\
* & -X & \left(E_{1} X+E_{2} Y\right)^{\mathrm{T}} & X & (\beta Y)^{\mathrm{T}} \\
* & * & -\gamma I & 0 & 0 \\
* & * & * & -Q^{-1} & 0 \\
* & * & * & * & -R^{-1}
\end{array}\right]+\left[\begin{array}{c}
B_{0} \\
0 \\
0 \\
0 \\
I
\end{array}\right] R_{0}\left[\begin{array}{c}
B_{0} \\
0 \\
0 \\
0 \\
I
\end{array}\right]^{\mathrm{T}}+\left[\begin{array}{c}
0 \\
Y^{T} \beta \\
0 \\
0 \\
0
\end{array}\right] R_{0}^{-1} \beta_{0}^{2}\left[\begin{array}{c}
0 \\
Y^{T} \beta \\
0 \\
0 \\
0
\end{array}\right]^{\mathrm{T}}<0
$$

It equals to

$$
\left[\begin{array}{ccccc}
\gamma D D^{\mathrm{T}}-X+B_{0} R_{0} B_{0}^{\mathrm{T}} & A_{0} X+B_{0} \beta Y & 0 & 0 & B_{0} R_{0} \\
* & -X+\beta Y^{\mathrm{T}} R_{0}^{-1} \beta_{0}^{2} \beta Y & \left(E_{1} X+E_{2} Y\right)^{\mathrm{T}} & X & (\beta Y)^{\mathrm{T}} \\
* & * & -\gamma I & 0 & 0 \\
* & * & * & -Q^{-1} & 0 \\
* & * & * & * & -R^{-1}+R_{0}
\end{array}\right]<0
$$

It follows from the Schur complement that the above inequality is equivalent to

$$
\left[\begin{array}{cccccc}
\gamma D D^{\mathrm{T}}-X+B_{0} R_{0} B_{0}^{\mathrm{T}} & A_{0} X+B_{0} \beta Y & 0 & 0 & 0 & B_{0} R_{0} \\
* & -R_{0} \beta_{0}^{-2} & \beta Y & \left(E_{1} X+E_{2} Y\right)^{\mathrm{T}} & X & (\beta Y)^{\mathrm{T}} \\
* & * & -X & 0 & 0 & 0 \\
* & * & * & -\gamma I & 0 & 0 \\
* & * & * & * & -Q^{-1} & 0 \\
* & * & * & * & * & -R^{-1}+R_{0}
\end{array}\right]<0
$$

We obtain the first condition of the optimization problem.

Following from the Schur complement, the second condition of the optimization is equivalent to $S>X^{-1}>0$, minimizing the $\operatorname{Trace}(S)$ will make $\operatorname{Trace}\left(X^{-1}\right)$ to be minimized, then the upper bound of the system performance index will be minimized.

\section{Conclusion}

In this paper, we have derived the existence condition for guaranteed cost control for a class of variable sampling period network control system with actuator failure. The optimal cost controller was obtained through LMI and Lyapunov stability theory.

\section{References}

[1] Gregory C. Walsh, Hong Ye, Linda G. Bushnell. "Stability analysis of networked control systems." Control Systems Technology IEEE Transactions 10 (2002): 438-446.

[2] Kun-Qiu Sun. "Guaranteed Cost Control Research for Networked Control Systems with Time-varying Sampling" Shenyang University of technology 2016. 
[3] FAN Jinrong. "Optimal cost control for networked control system with variable sampling period." Engineering Journal of Wuhan University 44. 6 (2011): 806-811.

[4] Kim Dong Sung, Young Sam Lee, Wook Hyun Kwon, Hong Seong Park. "Maximum allowable delay bounds of networked control systems." Control Engineering Practice 11. 11 (2003): 1301-1313.

[5] Miao Qi, Zhang Duanjin. "Recent Progression of Fault-Tolerant Control" Electrical Automation 2007, 9 (2).

[6] Huo Zhihong, Fang Huajing, Yan Guihuan. "Co-design for NCS robust fault-tolerant control" Transactions on Automatic Control 2004, 49 (2): 281-287.

[7] Zheng. Y. "Fault diagnosis and fault tolerant control of networked control system" Wuhan: Huazhong University of Science and Technology 2003.

[8] Gao Huijun, Wu Junli, Shi Peng. "Robust sampled-data $\mathrm{H} \infty$ control with stochastic sampling" Automatica 2009, 45 (7).

[9] FAN Jin-rong, FANG Hua-jing. "Robust fault tolerant control for networked control system with variable sampling period" Control Engineering of China 20. 5 (2013): 859-863.

[10] Yi Jianqiang. "BP neural network prediction-based variable-period sampling approach for networked control systems" Applied Mathematics \& Computation 185. 2 (2007): 976-988.

[11] Xie Guangming, Wang Long. "Stabilization of NCSs with time-varying transmission period." IEEE International Conference on Systems, Man and Cybernetics 2005: 3759-3763 Vol. 4.

[12] Yu-Long Wang, Guang-Hong Yang. "Hœ Controller design for network control system via active-varying sampling period method." ACTA AUTOMATICA SINICA 2008, 34 (7) 814-818.

[13] Li Wei, Y Li, and W Liu. "Robust Fault Tolerant Control for Networked Control Systems with Uncertain Disturbance." Mechatronics and Automation, 2007. ICMA 2007. International Conference on IEEE, 2007: 2813-2818.

[14] Li Yu. "An LMI approach to reliable guaranteed cost control of discrete-time systems with actuator failure." Applied Mathematics \& Computation 162.3 (2005): 1325-1331.

[15] Li Yu. "Robust control- Linear matrix inequality approach." Beijing: Tsinghua University Press 2002.

[16] C. Van Loan. "Computing integrals involving the matrix exponential." Automatic Control IEEE Transactions 23. 3 (1978): 395-404. 